\title{
Non-Invasive Transgastric Detection of Ultrasonic Doppler Signals from Canine Renal Vessels
}

\author{
Tadashi Inaba, Hiroki Watanabe, Hiroshi Ohe, \\ Hiroaki Miyashita, Hisashi Hayami and Makoto \\ WATANABE \\ Department of Urology, Kyoto Prefectural University of \\ Medicine, Kyoto 602
}

Inaba, T., Watanabe, H., Ohe, H., Miyashita, H., Hayami, H. and Watanabe, M. Non-Invasive Transgastric Detection of Ultrasonic Doppler Signals from Canine Renal Vessels. Tohoku J. exp. Med., 1987, 153 (1), 75-76We successfully obtained ultrasonic Doppler signals from canine renal vessels non-invasively by the transgastric approach. An outline of the method and newly developed transgastric probe is presented in this preliminary report.—— renal blood flow ; ultrasonic Doppler flowmeter; transgastric probe

\section{Methods}

An original transgastric probe was developed (Fig. 1). It is a convex probe, using a curved array transducer at the tip, which can visualize the organs and vessels through the gastric wall and can simultaneously detect blood flow in them. The effective length of the probe is $1 \mathrm{~m}$ and the outer diameter is $10.5 \mathrm{~mm}$. The tip is flexible and can be bent manually to a maximum of $120^{\circ}$ to the right or left.

The scanner is $40 \mathrm{~mm}$ in length, $12 \mathrm{~mm} \times 14 \mathrm{~mm}$ in cross-section, and provides an ultrasonic beam at a frequency of $5 \mathrm{MHz}$. The scanning direction is longitudinal for the probe, because the Doppler flow measurement system is included. The scanning angle is $34^{\circ}$ and the focal length is $32 \mathrm{~mm}$. Blood flow can be recorded by sound spectrogram or real-time two dimensional color flow mapping.

Experiments were carricarried out on five mongrel dogs anesthetized with pentobarbital. Each dog was fixed into the supine position and intubated. The probe was then inserted transesophageally into the stomach. When the kidney was visualized by setting the probe at a suitable position, the tip was bent about $60^{\circ}$ to $90^{\circ}$ to visualize the renal vessels. Blood flow signals from the renal vessels were recorded consecutively by sound spectrogram or color flow mapping.

\section{Results}

By this method all of the canine kidneys were visualized easily and clearly through the gastric wall. The sonogram of the canine kidney was almost the same as that of the human kidney. The canine renal veins could be visualized well by sonography but the renal arteries were too small to be detected. Detection of the Doppler signals from the renal artery proved difficult at first due to inexperience and we were only able to obtain signals

Received April 6, 1987; accepted for publication July 28, 1987. 

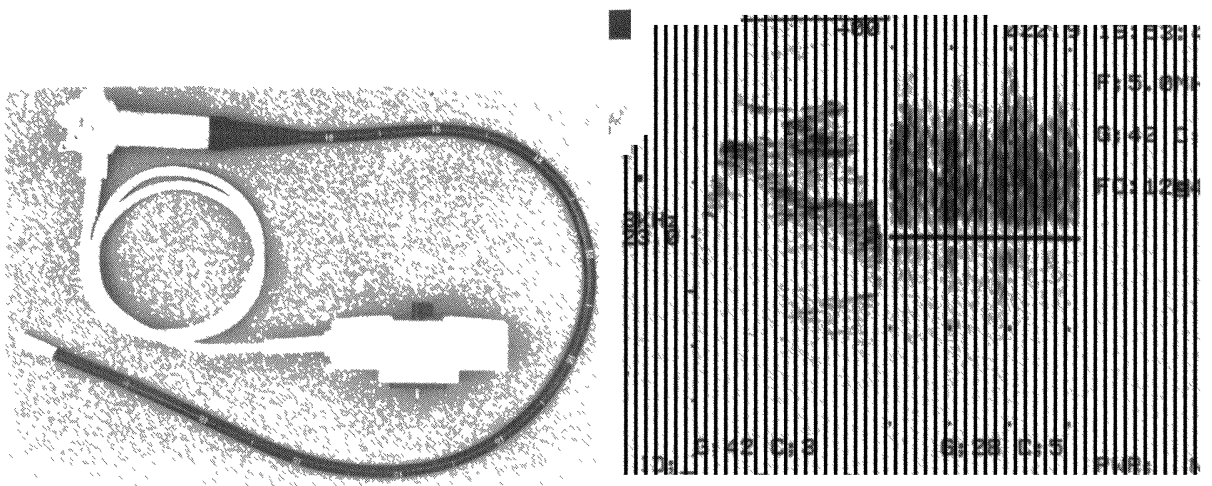

Fig. 1 (left). Originally developed transgastric probe for blood flow measurement in the renal artery.

Fig. 2 (right). Transgastrie sonogram of the canine kidney. The renal vein is clear and the central echoes can be easily identified (left). The spectrogram of the renal arterial blood flow shows periodic wave pattern (right).

from the small arteries in the kidney. However, for the last two dogs we managed to obtain enough signals from the left and right renal artery respectively with no differences in the detection rate. In color flow mapping blood flow in the artery was delineated as a strongly colored line. The pattern of blood flow from the artery was also represented by spectrogram, which showed a characteristic periodic wave. The maximum blood flow velocity in the left renal artery was calculated at $32 \mathrm{~cm} / \mathrm{sec}$ (Fig. 2).

\section{Discussion}

Non-invasive ultrasonic Doppler blood flow measurement of the renal artery has been tried since 1976 (Watanabe et al. 1976; Ohe et al. 1982). However, quantitative measurement of renal blood flow has so far been unsuccessful, because the renal vessels were located too deep in the body and were too small.

We developed an original transgastric probe, employing it for the canine kidney prior to clinical use, and successfully measured the maximum flow velocity in the renal artery. Since the canine renal artery was too small to be visualized by this method, the mean blood flow volume could not be calculated. However, this new endoscopic technique is expected to overcome several difficult current problems in clinical quantitative renal blood flow measurements.

\section{Acknowledgments}

This study was partly supported by Grant for Scientific Reseach from the Ministry of Education, Science and Culture, Japan.

\section{References}

1) Ohe, H., Watanabe, H. \& Inaba, T. (1982) The measurement of renal blood flow using pulsed Doppler with the UI Octoson. Ultrasound Med. Biol., 8, Suppl. 1, 143.

2) Watanabe, H., Saitoh, M., Igari, D., Tanahashi, Y.\& Harada, K. (1976) Non-invasive detection of ultrasonic Doppler signals from renal vessels. Tohoku J. exp. Med., 118, 393-394. 